\title{
La huerta escolar como estrategia pedagógica para fortalecer el aprendizaje ${ }^{1}$
}

\section{The school garden as a pedagogical strategy to strengthen learning}

DOI: http://dx.doi.org/10.17981/cultedusoc.9.3.2018.53

Artículo de investigación. Fecha de recepción: 15/06/2018. Fecha de aceptación: 27/11/2018

\author{
Yhonys Zambrano - Quintero²; \\ Concepción Rocha-Roja; Gala Flórez-Vanegas; Luz Nieto-Montaño; \\ Jaime Jiménez-Jiménez y Luz Núñez -Samnández ${ }^{3}$ \\ IED Oscar Pisciotti Numa, del Banco Magdalena (Colombia) \\ jonysrafael@hotmail.com
}

Para citar este artículo:

Zambrano-Quintero, Y., Rocha -Roja, C., Flórez-Vanegas, G., Nieto-Montaño, L., Jiménez-Jiménez, J. y Núñez -Samnández, L. (2018). La huerta escolar como estrategia pedagógica para fortalecer el aprendizaje. Cultura. Educación y Sociedad 9(3), 457-464. DOI: http:// dx.doi.org/10.17981/cultedusoc.9.3.2018.53

\section{Resumen}

El presente artículo se enfoca en la huerta escolar como una estrategia pedagogía que busca fortalecer la adquisición de aprendizajes de los estudiantes a través de la producción de alimentos de una forma natural y económica que aporten al desarrollo de una aprendizaje integral; el estudio se aborda desde una mirada cualitativa, con un alcance descriptivo, la unidad de análisis estuvo constituida por los estudiantes de quinto grado de la Institución Educativa Departamental (IED) Oscar Pisciotti Numa, donde se encontró que la mayoría de los estudiantes reconocen que a través de esta se pueden trabajar los diferentes temas de clases y adquirir mejores aprendizajes; lo que evidencia que la huerta, como estrategia de enseñanza aprendizaje resulta positiva pues permite aprender desde la práctica, en todas las áreas del saber.

Palabras clave: Huerta escolar, estrategia pedagógica, aprendizaje integral.

\section{Abstract}

This article focuses on the school garden as a pedagogy strategy that seeks to strengthen the acquisition of student learning through the production of food in a natural and economic way that contribute to the development of an integral learning; The study is approached from a qualitative perspective, with a descriptive scope. Participants were the fifth grade students of the Oscar Pisciotti Numa IED, where it was found that most students recognize that through this they can work different class topics and acquire better learning; which shows that the garden as a teaching-learning strategy is positive because it allows learning from practice, in all areas of knowledge

Keywords: School garden, pedagogical strategy, comprehensive learning.

\footnotetext{
1 Este artículo ha sido derivado del Programa de Fortalecimiento de la Cultura Ciudadana y Democrática CT+I a través de la IEP apoyada en TIC en el Departamento de Magdalena: proyecto CICLÓN

2 Líder del Grupo de investigación "Sembradores de Esperanza".

3 Docentes de la institución educativa departamental Oscar Pisciotti Numa, del Banco Magdalena, del grupo de investigación "Sembradores de Esperanza".
}

- The author; licensee Universidad de la Costa - CUC. 


\section{Introducción}

En el mundo globalizado de hoy, la cultura ambiental asociada con el aprendizaje en la escuela, han surgido cambios en la forma en la que se concibe la educación, donde el componente ambiental, surge como ente fundamental para generar cultura pro ambiental, pilar para el desarrollo de competencias ciudadanas y competencias científicas asociados a diferentes áreas del conocimiento.

Teniendo en cuenta los cambios presentes en el sistema educativo actual, se hace necesario idear, implementar e incorporar al currículo, herramientas pedagógicas innovadoras, que otorguen al mismo, flexibilidad, reciprocidad, carácter problematizador y participativo, donde el estudiante pueda vivenciar desde la realidad de su contexto la comprensión de saberes interdisciplinarios de las diferentes áreas de estudio, con el fin de que ejerzan un mayor compromiso y responsabilidad en su labor educativa; permitiendo con ello la convivencia y la reciprocidad del proceso educativo, obligando a orientarse a actividades de transformación de la educación, en búsqueda de mejoras en la transmisión de los saberes y en la apropiación de los mismos (Carrera, Bravo \& Marín 2013).

Observando la problemática existente en la institución educativa, enmarcada en la baja motivación, desinterés y bajo rendimiento académico de los estudiantes, se contempla como respuesta a esta situación la idealización y ejecución de proyectos productivos para dinamizar el proceso de enseñanza aprendizaje, desde la sostenibilidad, la innovación y el emprendimiento (Mújica, Marín, Smith y Lovera 2008); en este sentido se contempla la realización de una huerta escolar; como una estrategia pedagógica para estimular el aprendizaje de los estudiantes; pues esta implica una interacción directa con cada una de las áreas del saber educativo, fomentando en la comunidad un interés por realizar labores agroecológicas, propias del contexto y la realidad en la que viven los estudiantes. Es así como se concibe a la huerta escolar como recurso educativo que permite poner en práctica un aprendizaje activo y cooperativo, basado en un proceso de reflexión para dar respuesta a diferentes problemáticas dentro del contexto escolar, siendo ésta una estrategia para desarrollar actitudes y valores encaminados al fortalecimiento de competencias ciudadanas, la conservación y el cuidado del medio ambiente (Domínguez, 2011).

Teniendo en cuenta lo anterior, se busca mediante la implementación de huertas escolares fortalecer el aprendizaje de los estudiantes de cero a quinto grado del IED Oscar Pisciotti Numa, donde a su vez, el docente debe poseer las habilidades y competencias para el desarrollo de actividades pedagógicas que permitan a los estudiantes relacionarse directamente con su entorno, respetando la interdisciplinariedad como factor principal en los procesos del saber y el saber hacer. En este sentido, Vera (2015), señala que cobra importancia la huerta escolar como escenario para el desarrollo de competencias críticas e investigativas, que favorezcan la adquisición de nuevos saberes.

De acuerdo con Bastidas (2012) referido por Palacios (2016), la huerta escolar se concibe como estrategia que dinamiza el proceso de aprendizaje, que potencializa y desarrolla una enseñanza activa y dinámica, logrando trabajar desde un entorno vivo y físico, donde además de fortalecer el aprendizaje académico se fortalece un aprendizaje nutricional. Así mismo, plantea la huerta escolar como un escenario de participación e interacción entre la generación de saberes teóricos y prácticos de los niños y niñas. Es un espacio donde tanto niños como familia, desarrollan actividades productivas que generen beneficios a la institución, a la familia y a la comunidad en general. 
Es muy relevante en este aspecto el postulado de Mazzini (2012), quien sostiene que la huerta escolar además de ser una estrategia para construir aprendizajes significativos, es un espacios ejecutor, de acción y participación, que mejora la escuela y su entorno, que optimizan además la situación nutricional de los niños y niñas de la sede, las competencias científicas críticas y reflexivas de los estudiantes.

Mediante la aplicación de la huerta escolar, orientada desde la investigación como estrategia pedagógica, como elemento fundamental para garantizar la adquisición de nuevos conocimientos de manera dinámica, se busca indagar sobre los conocimientos y aptitudes que posee la comunidad educativa sobre las huertas escolares y su incidencia significativa en los procesos de aprendizaje y en la economía de sus beneficiarios, por lo que es necesario emplear técnicas y herramientas de recolección de la información como la observación y la encuesta para determinar el nivel de conocimiento que posee la población a estudiar (Cortés, García y Pérez, 2007).

\section{Las huertas escolares como escenarios de aprendizajes}

Se parte de la premisa de que la huerta escolar son pequeños espacios de las instituciones educativas cuyo objetivo primordial es generar destrezas y habilidades entorno al medio ambiente, donde el alumno llegue a comprender, reflexionar e indagar las relaciones entre las plantas, el ambiente y su relación con el entorno escolar, así mismo, que adquieran el conocimiento sobre la incidencia de nuestras actividades y acciones sobre el equilibrio del ambiente (Toloza, Osorio y Sandoval, 2012).

Palacios, Amud y Pérez (2016), sostienen que la huerta escolar es un recurso didáctico que puede utilizarse en todos los niveles educativos, permite convertir a los centros escolares en generadores de múltiples experiencias de aprendizaje, funciona como un incentivo vivo y cambiante, que desarrolla valores positivos. Promueve el conocimiento, experiencia, capacidades y habilidades prácticas, incluso aplicables fuera de la escuela, genera formación sobre nutrición y seguridad alimentaria, además de fomentar actitudes de responsabilidad y respeto hacia la naturaleza.

La importancia de trabajar con esta estrategia en la institución, radica en dinamizar la forma en que se imparten las clases, en innovar en el proceso de enseñanza, articulando los contenidos curriculares con experiencias prácticas propias del contexto, que despiertan el interés de los estudiantes por aprender, por asistir a la institución, en crear espacios para la crítica, la reflexión y la investigación apoyada en las TIC.

Según Toloza, Osorio y Sandoval (2012), dentro de los beneficios de trabajar desde las instituciones con las huertas se encuentran:

a. Una fuente de alimentos para mejorar la alimentación de los niños y su salud.

b. Un escenario para el aprendizaje (la naturaleza, la agricultura y la nutrición).

c. Un espacio para la indagación social y el esparcimiento, para desarrollar conductas o competencias en pro del cuidado del ambiente.

d. Un espacio de interacción, de participación, de reflexión y de construcción de conocimiento a partir de la experiencia práctica.

\section{La investigación como estrategia pedagógica}

Los autores, Manjarrés, Mejía y Ciprian (2011), señalan que la investigación como estrategia pedagógica (IEP), estudia la realidad en su contexto natural construye el espíritu científico utiliza variedad de instrumentos para recoger información como las entrevistas, las imágenes, observaciones, historias de vida, en los 
que se describen las rutinas y las situaciones problemáticas.

Además, se busca que a través del uso de la IEP, se fomente en la comunidad educativa la construcción colectiva, desarrollo de capacidades de cooperación y solidaridad con los niños, niñas, jóvenes y otras personas. Permitiendo desarrollar en las practicas pedagógicas unos ambientes innovadores de aprendizaje que despierten mayor interés y motivación en los estudiantes generando en ellos capacidades y habilidades en ciencias, tecnologías, dando viabilidad y aprovechamiento a los saberes y por tanto mejorar el desempeño académico de los mismos.

Es necesario señalar que la huerta escolar se articula con el Proyecto Educativo Institucional (PEI), a través del Proyecto Ambiental Escolar (PRAE), el cual se desarrolla tomando en cuenta las leyes ambientales que involucran la clasificación de basuras en la institución educativa (Cuadrado, Fernández, Monroy y Montaño, 2013).

Teniendo en cuenta lo anterior, se busca a través de la implementación de la huerta escolar mediada por la investigación como estrategia pedagógica, que los estudiantes se apropien del entorno escolar, aprendan a clasificar, categorizar, indagar, a generar opiniones críticas, que se apropien de los saberes para lograr favorecer el desarrollo integral del estudiante, propiciando un trabajo coordinado con la familia y la comunidad, en un proceso permanente de construcción y de conocimiento a partir de su realidad latente.

En este orden de ideas, cabe resaltar la gran importancia la transversalidad de las diversas áreas del saber, que se pueden trabajar a través de la huerta; pues a través de esta se pueden articular todos los contenidos académicos, creando espacio desde cada área para la investigación y experimentación (Toloza, Osorio y Sandoval, 2012).

\section{Metodología}

Teniendo en cuanta el objeto de la investigación, el presente proyecto de oriento desde una mirada Cualitativa, ya que busca comprender e interpretar la realidad de los fenómenos que se estudian en su contexto natural. (Martínez 2006). El alcance de la investigación es descriptivo, con un diseño transeccional, porque se recolectan datos en un solo momento, en un tiempo único.

\section{Participantes}

Se tomó como unidad de análisis un total de 30 estudiantes de quinto grado de la IED Oscar Pisciotti Numa, entre las edades de 11 y 12 años de edad, los cuales participaron de manera voluntaria, firmando sus padres el respectivo consentimiento informado.

\section{Instrumentos de recolección de información}

Para dar cumplimiento a los propósitos de la investigación se tomó como instrumento de recolección la entrevista estructurada. Al respecto Grawitz (2011), la define como la conversación de dos o más personas en un lugar determinado, para tratar un asunto o para recoger información específica de un tema en particular.

\section{Resultados}

A continuación se presentan los principales hallazgos derivados de la aplicación del instrumento, posterior a la observación que se realizó a los estudiantes en el área de ciencias naturales y su participación en clases, en el tema correspondiente a la huerta, en la cual se pudieron detectar los conocimientos que poseen los estudiantes de las hurtas escolares y su importancia en la implementación de esta como una estrategia pedagógica para fortalecer el aprendizaje. 
La entrevista estructurada contaba con los siguientes interrogantes

1. ¿Crees que el profesor enseñanza las clases de forma adecuada?

2. ¿Sabe usted que es una huerta escolar?

3. ¿Crees q a través de las huertas escolares su pueden trabajar diferentes temas de clases?

4. ¿Crees que el desarrollo de las huertas escolares es una buena herramienta para fortalecer el aprendizaje? Sí No__-__ ¿porque?

5. ¿Además de fortalecer el aprendizaje, qué otros beneficios puede generar la implementación de una huerta escolar?

6. ¿Le gustaría participar en la creación de la huerta escolar? Sí No

En cuanto a la pregunta número 1, la mayoría los estudiantes manifestaron que en algunas ocasiones tienden a aburrirse en las clases, debido a que el profesor solo da las clases con teorías, con la utilización de libros, dejando muchas tareas para la casa, no utiliza computadores, ni internet para que las clases sean más entretenidas.

De acuerdo a los hallazgos encontrados en la entrevista aplicada, se deduce de manera general que la atención y motivación de los estudiantes se ve afectada por las estrategias y actividades que utiliza el maestro para dar sus clases; estas situaciones presentadas son un punto de inicio para dar partida a la implementación de estrategias pedagógicas pertinentes que involucren de manera activa y participativa a los estudiantes, estrategias que propicien espacios de reflexión, indagación, análisis y critica.

En consecuencia se hace necesario promover estrategias que generen dinámicas de transformación en la metodología del docente, para innovar su proceso de enseñanza e invitar a la construcción de competencias científicas a los estudiantes, y sea un ente generador de cambios que impacten directamente en los procesos curriculares.
Con relación a las preguntas 2 , los estudiantes respondieron, que si saben que es una huerta, pues en el contexto en el cual está inmersa su comunidad, la actividad económica principal es la ganadería y la agricultura, dedicándose la mayoría de sus familias a las labores de campo. Sin embargo, estos manifiestan nunca haber tenido o conocido una huerta escolar, pero consideran que debe contemplar los mismos recursos de una huerta orgánica. De acuerdo a las respuestas, se deduce que los estudiantes han tenido el conocimiento y la experiencia de una huerta, ya sea en sus casas o en el campo.

Referente a las preguntas $\mathrm{N}^{\circ} 3,4$ y 5 , los estudiantes responden que a través de la huerta escolar se pueden trabajar diferentes asignaturas con diferentes temas, como el medio ambiente, los valores, temas de geografía, biología y español, ya que no solo se enseña a sembrar plantas si no que se participa, investiga y se aprende desde todas las áreas del saber. Así mismo, consideran que es una herramienta para fortalecer el aprendizaje, porque permite el trabajo en equipo, estimula las habilidades de indagación, de comprensión, de lectura, ayuda a través de la práctica a memorizar y a captar con mayor facilidad las clases y por tanto los aprendizajes. Además de ser una actividad divertida, trae como beneficios que se hacen más amigos, se aprende a respetar, a compartir, a generar sus propios alimentos y finalmente, que es propicia para empezar a utilizar herramientas tecnologías que ayudan a estudiar con más facilidad.

Analizando las respuestas de los estudiantes se evidencia la necesidad de implementar la huerta como una herramienta pedagógica para desarrollar y potencializar en los estudiantes capacidades científicas como la observación, exploración e investigación, generando cambios en la dinámica de aprendizaje y movilizando la aprehensión de nuevos saberes. Así mismo, a través de la experiencia práctica se hace 
posible el desarrollo de la capacidad de relacionarse, compartir, disfrutar, aprender y enriquecer su proceso de socialización, desarrollar una cultura emprendedora, trabajar en equipo, planificar actividades de manera conjunta, asumiendo responsabilidades y por tanto incrementando su motivación e interés por aprender (Vera 2015).

Con respecto a la pregunta $\mathrm{N}^{\circ} 6$, los estudiantes contestaron afirmativamente que querían participar y apoyar en la realización de la huerta, porque además de ayudarlos a aprender con mayor facilidad, es un espacio para compartir y trabajar con metodologias propias de su contexto social.

\section{Discusión}

Mediante los resultados obtenidos se hace pertinente y necesario fortalecer las estrategias a pedagogías implementadas; a fin de empoderar a la comunidad estudiantil, mejorar sus resultados académicos, y lograr incrementar el interés por las clases; pues se logró establecer que requieren actividades pedagógicas innovadoras que faciliten el proceso de aprendizaje.

En este sentido, cobra importancia la actitud y disposición de los maestros en adaptarse y cambiar la transmisión de contenidos desde el modelo tradicional de enseñanza, enmarcada en el aula de clase, a trabajar desde una metodología innovadora, a que se emplee la huerta escolar como estrategia didáctica, que facilita el aprendizaje de conceptos propios de las áreas del saber; que se constituye como un espacio de construcción de procesos investigativos, que permite desarrollar capacidades como la curiosidad, el deseo de conocer, indagar cuestionar, observar, criticar, reflexionar y solucionar problemas (Vera, 2015).

Estos resultados reflejan la necesidad de una intervención y un cambio en la metodología de enseñanza, cambios que le permita al estudiante comprender y conceptualizar las temáticas vistas de manera activa y práctica, donde se establezcan parámetros de participación constante y articulación con herramientas digitales y espacios de exploración. En este sentido, cobra importancia el trabajar con la huerta escolar como una herramienta pedagógica pues esta, como señala Maldonado y Pinzón (2016), facilita el desarrollo de una práctica educativa que permite enseñar desde el contexto real de los estudiantes, que aprendan desde su cotidianidad diferentes conceptos teóricos y prácticos necesarios para desarrollar un pensamiento crítico y analítico, requerido para fortalecer las capacidades de comprensión y aprensión de nuevos aprendizajes; así mismo, impulsa en la formación de valores y actitudes necesarias para un cambio hacia comportamientos más respetuosos con el medio ambiente.

Es de vital importancia que los docentes se apropien y empiecen a emplear otras técnicas y metodologías de enseñanza, que no solo fortalezcan los procesos académicos, sino que involucren aspectos emocionales y sociales que permitan el desarrollo integral del estudiante. La huerta escolar, en este sentido crea y despierta el interés de los estudiantes y agrado por permanecer en la institución, por aprender, por involucrarse en actividades pedagógicas experienciales.

Se reconoce y considera la huerta escolar como una estrategia pedagógica que genera expectativa en los estudiantes, que pretende impactar en la dinámica del proceso de enseñanza aprendizaje; pues tal como sostiene Moncada (2017), es una estrategia práctica en la cual los niños y las niñas van mejorando la escucha y la comunicación, permitiendo así desarrollar experiencias cooperativas a la hora de realizar un determinado proceso en la huerta, pues permite la puesta en marcha de razonamientos lógicos, capacidades de indagación y análisis, para dar solución a situaciones problemas propias del contexto. 
Para la implementación de esta estrategia pedagógica sea hace necesario involucrar a los padres de familia, pues en su gran mayoría trabajan en actividades relacionadas con el agro y aportarían sus conocimientos para lograr un diálogo de saberes entre el conocimiento empírico y el conocimiento científico. Así mismo, se le sugiere a las directivas de la institución brindar los elementos y el apoyo necesarios para la implementación de la huerta escolar, como estrategia pedagógica para que se sostenga y se establezca como una prioridad a nivel académico en la institución.

Finalmente, es importante lograr establecer la huerta escolar como una estrategia fundamental en todas las áreas del saber, dándole trascendencia desde las mallas curriculares, para otorgarle continuidad en el tiempo y lograr fortalecer desde los niveles más básicos de educación. En cuanto al aprendizaje, es una estrategia que potencializa habilidades sociales y científicas, necesarias para la vida escolar en la secundaria y a nivel profesional.

\section{Referencias}

Carrera, M., Bravo, O. y Marín, F. (2013). Visión transcompleja y socioopolítica del currículo universitario. Encuentro Educacional, 20(1)

Cortés, S., García, S. y Pérez, C. (2007). Estrategia pedagógica para favorecer la educación ambiental en primera infancia: manejo de residuos y recuperación de zonas verdes. [Tesis de especial]. Recuperado de http://repository.libertado$\mathrm{r}$ e s.e d u . c o / b i t s t r e a m / handle/11371/1224/cortessafira2017. pdf?sequence $=2$

Cuadrado, I., Fernández, I., Monroy, F. y Montaño, A. (2013). Estilos de aprendizaje del alumnado de Psicopedagogía y su implicación en el uso de las TIC y aprendizaje colaborativo. RED-Revista de Educación a Distancia, (35), 1-19.
Domínguez, I. (2011). El huerto ecológico en la Educación Secundaria. [Tesis magistral]. Universidad de Salamanca, Salamanca. Recuperado de https:// www.ucm.es/data/cont/media/www/ p a g - $050 / \mathrm{El} \% 20$ hu e r t o \% 20 ecol\%C 3\%B3gico\%20en\%20la\%20 educaci\%C3\%B3n\%20secundaria.\%20 TFM\%20Universidad\%20de\%20Salamanca\%20(2011-2012).\%20Esperanza\%20Dom\%C3\%ADnguez\%20Iralde. pdf

Grawitz, M. (2011). Métodos y técnicas de las ciencias sociales. México, D.F.: Fontana y Frey. Recuperado de http:// trabajosocialmazatlan.com/multimedia/files/InvestigacionPosgrado/Entrevista.pdf

Maldonado, D. y Pinzón, L. (2016). La huerta escolar como medio para el desarrollo de competencias cientificas y ciudadanas en estudiantes de un colegio público en Bogotá. [Tesis magistral]. Universidad de La Sabana, Bogotá, D.C.

Manjarrés, M., Mejía, R. y Ciprian, J. (2011). Manual de apoyo a la gestión y a la construcción del Programa Ondas. Bogotá, D.C.: Colciencias.

Mazzini, R. (Octubre 12, 2012). Secuencias didácticas y huerta escolar. Recuperado de http:// arconrecursosisfd119pep.blogspot. com.co/p/blog-page.html

Moncada, S. (2017). La huerta escolar agroecológica como ambiente de aprendizaje colaborativo en el Colegio Monseñor Ramón Arcila del Carmen de Viboral. [Tesis pregrado]. Universidad de Antioquia-Seccional Oriente, El Carmen de Viboral.

Mujica, M., Marín, F., Smith, H. y Lovera, M. (2008). Municipio Innovador: Un modelo para el desarrollo local sostenible. Multiciencias. 8. 130-136. 
Palacios, J., Amud, N. y Mendoza, D. (2016). Implementación de huertas escolares como estrategia de enseñanza-aprendizaje de la biología de grado sexto en la Institución Educativa Agrícola de Urabá del municipio de Chigorodó y de grado séptimo de la Institución Educativa Rural Zapata, de Necoclí, departamento de Antioquia. [Tesis magistral]. Universidad Pontificia Bolivariana, Medellín.

Quesada, D. (2009). Cuestiones de Teoría del Conocimiento. Madrid: Tecnos.

Spina, M., Rohvein, C., Urrutia, S., Roark, G., Paravié, D. y Corres, G. (2016). Aplicación del modelo SCOR en pymes metalmecánicas de Olavarría. INGE CUC, 12(2), 50-57. https://doi.org/10.17981/ingecuc.12.2.2016.05
Toloza, Y., Osorio, E. y Sandoval, S. (2012). Proyecto ecológico huerta escolar [Mensaje en un blog]. Recuperado de http://escuelaruralelrosario.blogspot.com.co/2012/11/proyecto-ecologico-huerta-escolar.html .

Vera, J. (2015). La huerta escolar como estrategia didáctica para el desarrollo de competencias científicas en la institución educativa maestro Pedro $\mathrm{Nel}$ Gómez. [Tesis magistral]. Universidad Nacional de Colombia, Medellín. 